\title{
Images denoising in case impulse noise using spline approximation
}

\author{
Dmitriy A. Bezuglov ${ }^{1, *}$, Viacheslav V. Voronin ${ }^{2}$, and Vladimir A. Krutov ${ }^{3}$ \\ ${ }^{1}$ Rostov Branch of Russian Customs Academy, 344002 Rostov-on-Don Budennovskiy 20, Russia \\ ${ }^{2}$ Don State Technical University, 344000 Rostov-on-Don Gagarina 1, Russia \\ ${ }^{3} \mathrm{NPO}$ scientific and production association "Special Equipment and Communication", 344092 \\ Rostov-on-Don Dobrovolskogo 11/7, Russia
}

\begin{abstract}
Analytical equations of a new spline approximation method for filtering impulse noise in images are obtained. The proposed method differs from the known ones: when filtering images, one-dimensional sequential spline functions are used for direct and inverse transformations, and the processing is performed in rows and columns. In this work, experimental studies based on computer simulation using special test images on the background of impulse noise were conducted. Experimental studies have shown the operability and high efficiency of the developed method, which allow to improve the quality of image filtering by up to $10 \mathrm{~dB}$. In this case, the properties of spline functions make it possible to abandon the use of various masks, that is, to abandon inefficient linear methods of image filtering. The method can be used to create digital image processing systems in the industry, to create autonomous robots, under observation conditions that complicate the registration process, and in the absence of a priori information about the form of background noise.
\end{abstract}

\section{Introduction}

Ensuring the necessary requirements for modern vision systems for solving problems of high complexity, coupled with high variability of the working scene, heterogeneity of objects, interference, etc., is directly related to the task of increasing the efficiency of the digital image processing process recorded by sensors of such systems. In particular, one of the problems of applying digital image processing in a priori unknown observation conditions is the presence of noise, including pulsed, due to various factors, such as defects in the recording system, environmental influences, etc [1].

Recently, the onrush of microprocessor technology creates conditions for the emergence of new applications and the expansion and existing spectrum of problems solved using technical vision systems, which indicates the relevance of research in improving the efficiency and sustainability of digital image processing methods. To solve such problems, it is necessary to create appropriate mathematical methods for image processing, as well as software for specific tasks $[2,3]$.

\footnotetext{
*Corresponding author: bezuglovda@mail.ru
} 
Thus, the task of developing a scientific and methodological apparatus for digital image filtering in vision systems using spline approximation methods has not been adequately solved at the present time and is undoubtedly relevant.

One of the trends in the development of modern information technologies is the development of methods and algorithms for image analysis against the noise background of registration. Without the ability to effectively solve problems of this kind it is impossible to talk about the creation of appropriate image processing systems. Successful implementation of the prospects for the development of information and telecommunication technologies is largely based on the achievements of digital image processing, designed to solve the problems of forming, receiving, transmitting and processing information in real time. The implementation of complex digital image processing algorithms requires the use of effective basic algorithms (spectral analysis, filtering, compression, synthesis and identification), economically using the appropriate technological resources [4, 5].

The solution of the problem of image filtering is used in industry to create autonomous robots, and image analysis systems in difficult observation conditions, for the effects of various interfering factors that complicate the registration process and in the absence of a priori information about the form of background noise. This means that the methods and algorithms for processing information from image sensors must take into account the presence of noise of different nature associated with the registration of images in real systems, including impulse noise [6]. As a rule, a priori knowledge of the characteristics of distorting interference is required for constructing image filtering methods.

The purpose of the study is the development and analysis of a new method of digital filtering of images registered in the background noise using spline approximation.

\section{Images denoising using spline approximation}

The mathematical apparatus of spline approximation and cubic B-splines are effectively used in modeling a large number of different information processes. The equation for a parabolic B-spline is as follows:

$$
F_{c}(x)=\sum_{i=0}^{N+1} b_{i} B_{3}^{\mathrm{i}}\left(\chi_{i 3}\right), \quad \chi_{i 3}=\frac{1}{h}\left(x-x_{i+\frac{n+1}{2}}\right),
$$

where $b_{i}$ - spline coefficients; $x_{i+\frac{n+1}{2}}$ - coordinates of the middle of the B-spline support; $n$ spline order, $h$ - grid size.

$$
\begin{gathered}
B_{3}^{i}\left(\chi_{i 3}\right)=\frac{1}{6}\left(2+\chi_{i 3}\right)^{3} B_{0}^{i}+\left(\frac{2}{3}-\chi_{\mathrm{i} 3}^{2}-\frac{\chi_{i 3}^{3}}{2}\right) B_{3}^{i+1}+ \\
+\left(\frac{2}{3}-\chi_{\mathrm{i} 3}^{2}+\frac{\chi_{i 3}^{3}}{2}\right) B_{3}^{i+2}+\frac{1}{6}\left(2-\chi_{\mathrm{i} 3}\right)^{3} \mathrm{~B}_{0}^{i+3} \\
B_{0}^{i}= \begin{cases}1 \text { where } & x \in\left[x_{i} ; x_{i+1}\right] . \\
0 \text { where } & x \notin\left[x_{i} ; x_{i+1}\right]\end{cases}
\end{gathered}
$$

The smoothing problem will be solved by minimizing the functional of the form:

$$
J=\rho \int_{a}^{b}\left|F^{\prime \prime}(x)\right|^{2} d x+\sum_{i=1}^{N}\left(F_{u}\left(x_{i}\right)-F_{c}\left(x_{i}\right)\right)^{2},
$$


where $\rho$-smoothing coefficient; $F_{u}\left(x_{i}\right)$ - the value of the smoothing function in the grid nodes; $F_{c}\left(x_{i}\right)$ - spline value at point $x_{i} ;\left|F^{\prime \prime}(x)\right|$ - module of the second derivative of the spline.

Consider the interval $\left[x_{i} ; x_{i+1}\right]$ and realize the binding of the spline coefficients to the middle of the corresponding support. We introduce the normalized coordinate for the spline, equal to

$$
\chi=\frac{x-x_{i}}{h} .
$$

After simple arithmetic transformations, groupings concerning the spline coefficients and substitutions, we obtain an analytical equation for the spline in the form:

$$
\begin{aligned}
F_{c}(\chi) & =\sum_{i=1}^{N-1}\left(\frac{1}{6} \chi^{3}\left(b_{i+2}-3 b_{i+1}+3 b_{i}-b_{i-1}\right)+\frac{1}{2} \chi^{2}\left(b_{i+1}-2 b_{i}+b_{i-1}\right)+\right. \\
& \left.+\frac{1}{2} \chi\left(b_{i+1}-b_{i-1}\right)+\frac{1}{6}\left(b_{i+1}+4 b_{i}+b_{i-1}\right)\right) .
\end{aligned}
$$

Thus, the functional (3) is written in the form:

$$
J=\rho \sum_{i=1}^{N-1} \int_{x_{i}}^{x_{i+1}}\left|F^{\prime \prime}(x)\right|^{2} d x+\sum_{i=1}^{N}\left[F_{u}\left(x_{i}\right)-\frac{1}{6}\left(b_{i+1}+4 b_{i}+b_{i-1}\right)\right]^{2} .
$$

To find the coefficients of the spline (4) bringing the minimum of functional (5), we calculate the partial derivatives $\frac{\partial J}{\partial b_{j}} \quad(i=0 ; \overline{N+1})$ and equate them to zero. As a result of this operation, we obtain a system of $N+2$ linear equations whose matrix form has the form:

$$
\frac{1}{6}(a \cdot b)=z
$$

The matrix of coefficients of the system (6) has a seven-diagonal form and is well conditioned. Solving this system by one of the known methods, we find the values of the desired coefficients of the splines.

The mathematical equation for filtering images on the background of impulse noise regarding B-splines will be as follows:

$$
\begin{gathered}
F_{x}(\chi)=\sum_{i=1}^{N-1}\left(\frac{1}{6} \chi^{3}\left(b_{i+2}^{\mathrm{n}}-3 b_{i+1}^{n}+3 b_{i}^{n}-b_{i-1}^{n}\right)+\frac{1}{2} \chi^{2}\left(b_{i+1}^{n}-2 b_{i}^{n}+b_{i-1}^{n}\right)+\right. \\
\left.+\frac{1}{2} \chi\left(b_{i+1}^{n}-b_{i-1}^{n}\right)+\frac{1}{6}\left(b_{i+1}^{n}+4 b_{i}^{n}+b_{i-1}^{n}\right)\right) \\
F_{y}(\gamma)=\sum_{j=1}^{M-1}\left(\frac{1}{6} \gamma^{3}\left(b_{j+2}^{\mathrm{m}}-3 b_{j+1}^{\mathrm{m}}+3 b_{j}^{\mathrm{m}}-b_{j-1}^{\mathrm{m}}\right)+\frac{1}{2} \gamma^{2}\left(b_{j+1}^{\mathrm{m}}-2 b_{j}^{\mathrm{m}}+b_{j-1}^{\mathrm{m}}\right)+\right. \\
\left.+\frac{1}{2} \gamma\left(b_{j+1}^{\mathrm{m}}-b_{j-1}^{\mathrm{m}}\right)+\frac{1}{6}\left(b_{j+1}^{\mathrm{m}}+4 b_{j}^{m}+b_{j-1}^{m}\right)\right) \\
\bar{S}(i, j)=\sqrt{F_{x}^{2}+F_{y}^{2}}
\end{gathered}
$$

Thus, the proposed new method of filtering digital grayscale images suggests the following operations:

1. The calculation of spline approximation coefficients by normalized smoothing onedimensional cubic B-splines for all rows and columns of a digital grayscale image $S$ (6). 
2. Calculation of two gray-level arrays at each point of the image by the inverse transform, in which the analytical spline functions of the inverse transform are used as the core of the transform by the corresponding rows and columns (7).

3. Calculating the brightness at each point of the image by calculating the average brightness of the image (7).

Evaluation of filtration efficiency used as quantitative criteria in the following:

1. Root-mean-square deviation $\mathrm{e}_{r m s d}$ :

$$
e_{r m a d}=\sqrt{\frac{1}{M N} \sum_{i=0}^{N-1} \sum_{j=0}^{M-1}\left(M K_{i j}-M K_{i j}\right)^{2}}
$$

where $M K$ - test image, $M K$ - image obtained by the proposed method.

2. Peak to signal-noise ratio $S N R$ :

$$
\mu=\frac{1}{M N} \sum_{i=0}^{N-1} \sum_{j=0}^{M-1}\left(M \hat{K}_{i j}\right)^{2} S N R=\frac{255-\mu}{\sqrt{\frac{1}{M N} \sum_{i=0}^{N-1} \sum_{j=0}^{M-1}\left(\hat{M K_{i j}}-\mu\right)^{2}}},
$$

where $\mu$ - average value $\hat{M} K_{i j}$.

3. Peak to signal-noise ratio $S N R F$ (using in the calculations of the root-mean-square deviation):

$$
\begin{gathered}
S N R F=\frac{255-\mu}{\sigma_{\text {background }}} \\
\sigma_{\text {background }}=\sqrt{\frac{1}{\left(N_{\text {background }}\right)^{2}} \sum_{i=n_{1}}^{n_{1}+N_{\text {background }}} \sum_{j=m_{1}+N_{\text {background }}}\left(\hat{M K_{i j}}-\mu_{\text {background }}\right)^{2}} \\
\mu_{\text {background }}=\frac{1}{\left(N_{\text {background }}\right)^{2}} \sum_{i=n_{1}}^{n_{1}+N_{\text {background }}} \sum_{j=m_{1}}^{m_{1}+N_{\text {background }}}\left(\hat{M K_{i j}}\right)
\end{gathered}
$$

where: $\sigma_{\text {background }}$ - root-mean-square deviation of background; $\mu_{\text {background }}-$ mean background; $n_{1}, m_{1}$ - coordinates of the selected background area size $N_{\text {background }} \mathrm{X} N_{\text {background }}$ on the test image $\hat{M K}_{i j}$.

\section{Experimental results}

The order of the computational experiment was as follows. We used the original image Cameraman. In the future, all the resulting images will be compared with it.

Then the image of Cameraman was exposed to 2 types of noise. For example, the figures show images that have been exposed to impulse noise "broken pixels" with probability $p=0.5$ (Fig. 1), impulse noise "salt-pepper" with probability $p=0.5$ (Fig. 2).

Figures 3 and 4 show the results of Gauss filter processing of the images presented in Figures 1 and 2, respectively. Figures 5 and 6 show the results of image processing, presented in Figures 2 and 3, respectively, using the spline approximation method.

The results of the computational experiment as applied to the "broken pixels" type of pulse noise with different noise probabilities $p=0.3,0.5,0.7$ and smoothing ratio values 
$\rho=5,10,50,100$ are shown in table 1 . Similar results related to the impulse noise "salt-pepper" are presented in Table 2.

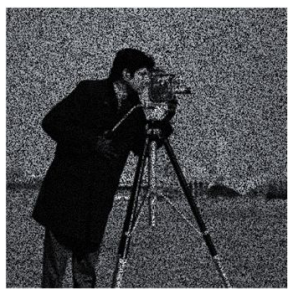

Fig. 1. The result of exposure to impulse noise "broken pixels".

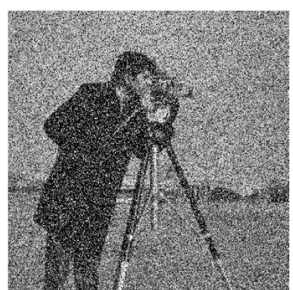

Fig. 2. The result of exposure to impulse noise "salt-pepper".

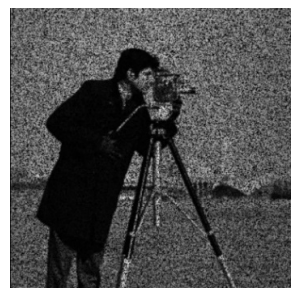

Fig. 3. The result of processing the Gauss filter image 2 .

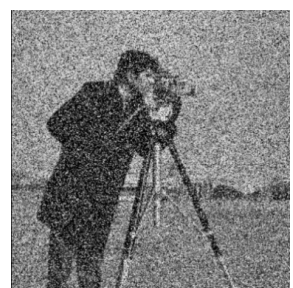

Fig. 4. The result of processing the filter Gauss image 3.

Table 1. The results of the computational experiment as applied to the "broken pixels" noise

\begin{tabular}{|c|c|c|c|c|}
\hline \multirow[b]{2}{*}{ Probability of noise } & \multicolumn{4}{|c|}{ Smoothing coefficient of spline functions } \\
\hline & 5 & 10 & 50 & 100 \\
\hline 0.3 & 1.8 & 2.01 & 2.22 & 2.23 \\
\hline 0.5 & 2.03 & 2.31 & 2.6 & 2.64 \\
\hline 0.7 & 2.13 & 2.42 & 2.75 & 2.79 \\
\hline \multicolumn{5}{|c|}{ Criterion 2, The advantage for the peak to signal-noise ratio $S N R, \mathrm{~dB}$} \\
\hline \multirow[b]{2}{*}{ Probability of noise } & \multicolumn{4}{|c|}{ Smoothing coefficient of spline functions } \\
\hline & 5 & 10 & 50 & 100 \\
\hline 0,3 & 3.47 & 4.09 & 5.22 & 5.48 \\
\hline 0,5 & 3.76 & 4.57 & 5.87 & 6.32 \\
\hline 0,7 & 3.97 & 4.85 & 6.45 & 6.87 \\
\hline \multicolumn{5}{|c|}{$\begin{array}{l}\text { Criterion 3, The advantage for the signal-noise ratio of the root-mean-square deviation of background } S N R F \text {, } \\
\mathrm{dB}\end{array}$} \\
\hline \multirow[b]{2}{*}{ Probability of noise } & \multicolumn{4}{|c|}{ Smoothing coefficient of spline functions } \\
\hline & 5 & 10 & 50 & 100 \\
\hline 0,3 & 5.23 & 6.08 & 8.11 & 8.9 \\
\hline 0,5 & 5.41 & 6.34 & 8.15 & 8.94 \\
\hline 0.7 & 5.63 & 6.65 & 8.73 & 9.4 \\
\hline
\end{tabular}

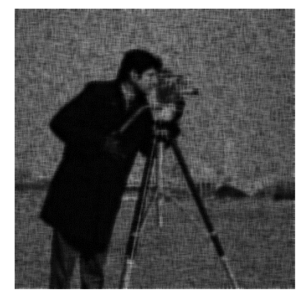

Fig. 5. The result of processing using the spline approximation method of the image of Cameraman.

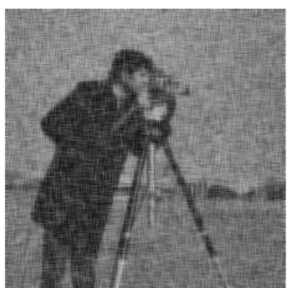

Fig. 6. The result of processing using the spline approximation method of the image of Cameraman.

\section{Conclusion}

An original method of image filtering based on a mathematical apparatus by spline approximation by normalized smoothing one-dimensional cubic B-splines was proposed and implemented. This method differs from the well-known ones in that when filtering images one-dimensional sequentially spline functions are used for direct and inverse transformations, and the processing is performed in rows and columns. Software 
implementation of the proposed method allows you to automate image processing and expand the possibilities of conducting research to create promising systems for their processing.

Experimental studies based on computer simulation using special test images in the background of impulse noise have been carried out. Experimental studies have shown the operability and high efficiency of the developed method, which allow to improve the quality of image filtering by up to $10 \mathrm{~dB}$.

Table 2. The results of the computational experiment as applied to the "salt-pepper" noise

\begin{tabular}{|c|c|c|c|c|}
\hline \multirow[b]{2}{*}{ Probability of noise } & \multicolumn{4}{|c|}{ Smoothing coefficient of spline functions } \\
\hline & 5 & 10 & 50 & 100 \\
\hline 0.3 & 2.51 & 2.69 & 2.83 & 2.81 \\
\hline 0.5 & 2.95 & 3.23 & 3.5 & 3.53 \\
\hline 0.7 & 3.22 & 3.55 & 3.92 & 3.96 \\
\hline \multicolumn{5}{|c|}{ Criterion 2, The advantage for the peak to signal-noise ratio $S N R, \mathrm{~dB}$} \\
\hline \multirow[b]{2}{*}{ Probability of noise } & \multicolumn{4}{|c|}{ Smoothing coefficient of spline functions } \\
\hline & 5 & 10 & 50 & 100 \\
\hline 0.3 & 5.13 & 5.77 & 6.57 & 6.56 \\
\hline 0.5 & 5.4 & 6.15 & 7.16 & 7.44 \\
\hline 0.7 & 5.65 & 6.46 & 7.78 & 8.07 \\
\hline \multicolumn{5}{|c|}{$\begin{array}{l}\text { Criterion 3, The advantage for the signal-noise ratio of the root-mean-square deviation of } \\
\text { background } S N R F, \mathrm{~dB}\end{array}$} \\
\hline \multirow[b]{2}{*}{ Probability of noise } & \multicolumn{4}{|c|}{ Smoothing coefficient of spline functions } \\
\hline & 5 & 10 & 50 & 100 \\
\hline 0.2 & 6.05 & 6.93 & 8.7 & 9.59 \\
\hline 0.3 & 6.17 & 7.08 & 9 & 9.69 \\
\hline 0.5 & 6.07 & 7.05 & 8.99 & 9.79 \\
\hline 0.7 & 6.12 & 7.14 & 9.32 & 10.12 \\
\hline
\end{tabular}

This work was supported by Russian Ministry of Education and Science in accordance to the Government Decree № 218 from April 9, 2010 (project number № 074-11-2018-013 from May 31, 2018 (03.G25.31.0284)).

\section{Reference}

1. D.A. Bezuglov, A.P. Kuzin, V.V. Voronin, Proc. SPIE 9869, Mobile Multimedia/Image Processing, Security, and Applications, 9869, 98690Q (2016)

2. D.A. Bezuglov, Y.D. Bezuglov, S.A. Shvidchenko, WSCG 2014 Conference on Computer Graphics, Visualization and Computer Vision, 15 (2014)

3. Z. Gordić, C. Ongaro, Serbian journal of electrical engineering, 13 (1), 9 (2016)

4. A. Zeković, I. Reljin, Serbian journal of electrical engineering, 11 (1), 25 (2014)

5. V. Voronin, V. Marchuk, D. Bezuglov, M. Butakova, Proceedings of the First International Scientific Conference "Intelligent Information Technologies for Industry" (IITI'16). Advances in Intelligent Systems and Computing, 451, 163 (2016)

6. V. Krutov, D. Bezuglov, V. Voronin, Serbian Journal of Electrical Engineering, 14 (3), 387 (2017) 